\section{Prolactin for spinal muscular atrophy}

\section{By Kai-Jye Lou, Staff Writer}

Ottawa researchers have shown that the peptide hormone prolactin increased survival of mice with spinal muscular atrophy. ${ }^{1}$ The researchers now plan to optimize their dosing protocol to see if they can further boost efficacy. The findings suggest that recombinant human prolactin, which Sanofi's Genzyme Corp. unit had shelved, could have potential in spinal muscular atrophy.

Spinal muscular atrophy (SMA) is an autosomal recessive disorder caused by abnormally low levels of the survival of motor neuron (SMN) protein in motor neurons, which lead to neurological dysfunction and muscle atrophy.

Low SMN levels result from a deficiency in the survival of motor neuron 1 telomeric (SMN1) gene, which is one of two nearly identical genes that encode SMN. This deficiency leaves only the other gene,

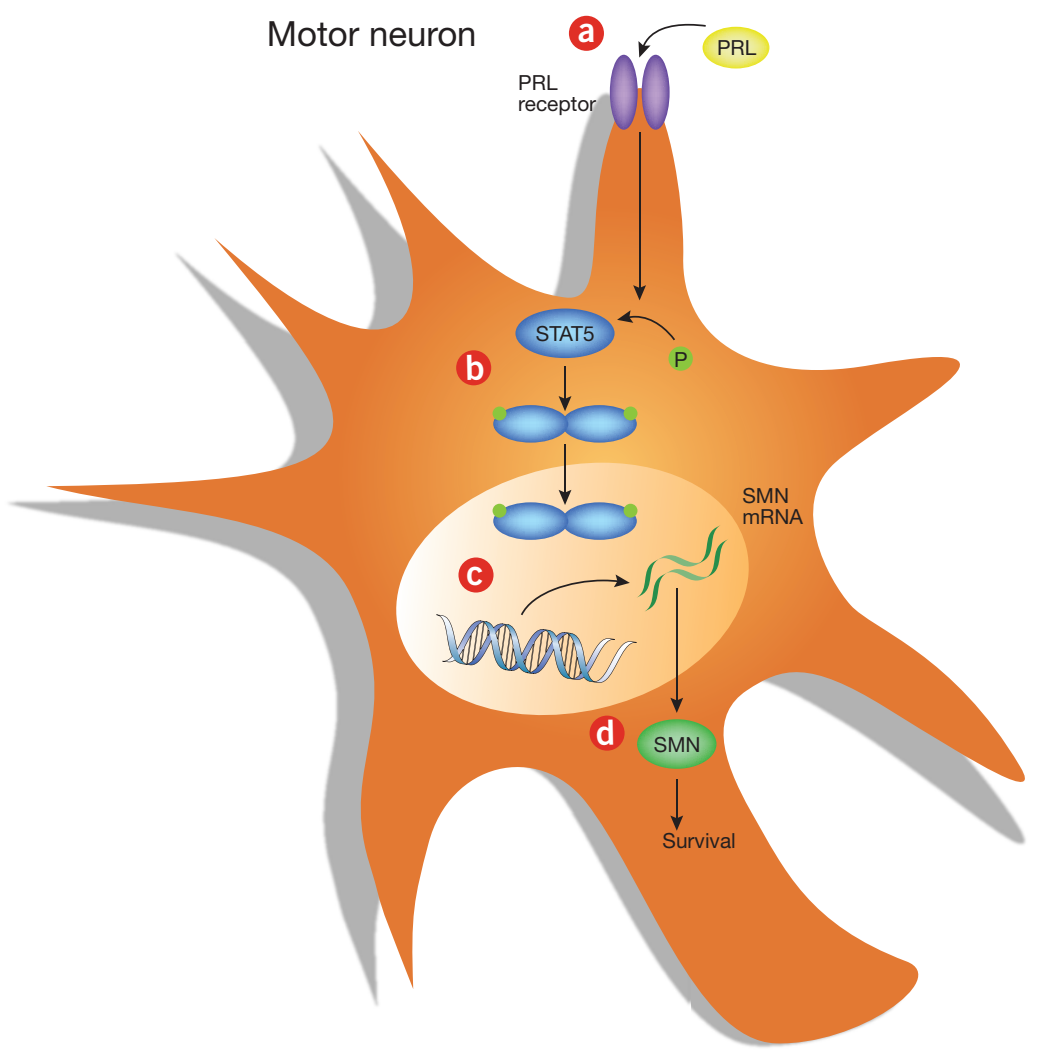

survival of motor neuron 2 centromeric (SMN2; SMNC), to produce protein. However, most mRNA transcripts from SMN2 encode an unstable and rapidly degraded variant of SMN.

In 2007, researchers from Academia Sinica showed that several classes of compounds, such as histone deacetylase (HDAC) inhibitors and chemotherapies, increased expression of SMN by SMN2 by activating signal transducer and activator of transcription 5 (STAT5). ${ }^{2}$

Researchers from the Children's Hospital of Eastern Ontario Research Institute and the University of Ottawa hypothesized that other activators of STAT5 not tested by the Academia Sinica group might also increase production of SMN in motor neurons. The team focused on the STAT5 activator prolactin (PRL). ${ }^{3,4}$ (See Figure 1, "Promoting motor neuron survival with prolactin.") Because recombinant human prolactin has been evaluated in previous investigator-led clinical trials for its ability to promote lactation, the authors thought this hormone could have safety advantages over other known STAT5 activators, such as HDAC inhibitors.

In a mouse model of severe SMA, daily intraperitoneal injections of prolactin increased Smn mRNA and protein levels in brain and spinal cord tissues compared with saline injections. The treated mice also showed greater motor function and had median survival of 21 days compared with 14 days for saline-treated controls $(p<0.0001)$.

Results were published in The Journal of Clinical Investigation.

"We see as much as an eightfold increase in SMN levels in this severe SMA mouse model," said Alexander MacKenzie, corresponding author on the paper and a principal investigator at the Children's Hospital of Eastern Ontario Research Institute. "In contrast, HDAC inhibitors like trichostatin A and valproic acid typically get about threefold increases."

Kathryn Swoboda, director of the Pediatric Motor Disorders Research Program and an associate professor in the Department of Neurology at The University of Utah School of Medicine, thinks prolactin-induced SMN upregulation could be even more potent in humans because the SMN2 promoter has more STAT5-binding sites than its mouse counterpart.

Figure 1. Promoting motor neuron survival with prolactin. As reported by Farooq et al., the peptide hormone prolactin (PRL) promotes survival of motor neuron (SMN) protein expression and increases overall survival in mouse models of spinal muscular atrophy (SMA).

$P R L$ activates the $P R L$ receptor [a], which promotes the phosphorylation $(P)$ and activation of signal transducer and activator of transcription 5 (STAT5) [b]. The activated transcription factor dimerizes and translocates into the nucleus, where it promotes SMN gene expression [c]. The resulting increase in SMN protein levels promotes motor neuron survival [d]. 
Swoboda noted that because prolactin has already been tested in human subjects and has generally had a good safety profile, it is an attractive candidate to consider for clinical trials in the immediate future as the community waits for other promising treatment approaches, such as antisense oligonucleotide therapies.

Another treatment approach being pursued for SMA is gene therapy.

A group from The Ohio State University and The Research Institute at Nationwide Children's Hospital showed last year that i.v. SMN gene therapy increased survival to more than 250 days from a median of 15.5 days for a control vector in the same mouse model that the Ottawa group used. ${ }^{5}$

The up to $70 \%$ increase in survival from prolactin is higher than historical rodent data for HDAC inhibitors, which have shown a $20 \%-30 \%$ increase.

\section{Getting optimal}

MacKenzie said his group is now trying to optimize the dosing protocol for prolactin to achieve further improvements in its effects.

"If we can fine tune our dosing protocol to get twice the survival benefit we see now, we could be in a position to move prolactin into clinical testing," he said. "We will also need to determine to what extent a robust prolactin therapy will affect established SMA. We will also need to determine whether prolactin therapy could stabilize the disease and whether it can improve the condition."

In the study published in JCI, the mice received prolactin at postnatal day 1 , prior to the establishment of SMA.

Charlotte Sumner, an assistant professor of neurology and neuroscience at The Johns Hopkins University School of Medicine, wanted prolactin tested in SMA models that have a milder phenotype. "It will also be important to show that prolactin-induced SMN expression improves motor neuron numbers and neuromuscular junction function," she added.

Swoboda said it will be important to develop an effective screen to identify newborns with SMA because upregulating SMN after most neurons have died is unlikely to yield meaningful benefits. She added that the impact of chronic delivery of prolactin on a child's endocrine system will need to be assessed.

The findings in the paper are not patented. Should prolactin show promise in future studies, MacKenzie said the group will need to seek development rights to the peptide from Genzyme.

Genzyme previously evaluated recombinant human prolactin as a nutraceutical to help boost the immune system but has since shelved the compound. The company, which has multiple issued patents covering recombinant human prolactin, did not respond to requests for comment.

The prolactin used in the JCI study was purchased from research reagent company Cedarlane Laboratories Ltd.

Lou, K.-J. SciBX 4(32); doi:10.1038/scibx.2011.896

Published online Aug. 18, 2011

\section{REFERENCES}

1. Farooq, F. et al. J. Clin. Invest.; published online July 25, 2011; doi:10.1172/JCl46276

Contact: Alex MacKenzie, Children's Hospital of Eastern Ontario Research Institute, Ottawa, Ontario, Canada e-mail: mackenzie@cheo.on.ca

2. Ting, C.-H. et al. Hum. Mol. Genet. 16, 499-514 (2007)

3. Goffin, V. et al. Genet. Anal. 15, 189-201 (1999)

4. Olazabal, I. et al. Mol. Endocrinol. 14, 564-575 (2000)

5. Foust, K.D. et al. Nat. Biotechnol. 28, 271-274 (2010)

\section{COMPANIES AND INSTITUTIONS MENTIONED}

Academia Sinica, Taipei, Taiwan

Cedarlane Laboratories Ltd., Burlington, Ontario, Canada Children's Hospital of Eastern Ontario Research Institute, Ottawa, Ontario, Canada

The Johns Hopkins University School of Medicine, Baltimore, Md. Genzyme Corp., Cambridge, Mass.

The Ohio State University, Columbus, Ohio

The Research Institute at Nationwide Children's Hospital, Columbus, Ohio

Sanofi (Euronext:SAN; NYSE:SNY), Paris, France

University of Ottawa, Ottawa, Ontario, Canada

The University of Utah School of Medicine, Salt Lake City, Utah 